\title{
Pros and Cons of Inhaled Corticosteroids Withdrawal in Chronic Obstructive Pulmonary Disease
}

\author{
Andrea Rossi, MD, FERS and Erika Zanardi, MD
}

Study Center of AIPO (Italian Association of Hospital Pulmonologists), Milano, Italy

\section{ABSTRACT}

Inhaled corticosteroids (ICS) are widely prescribed in chronic obstructive pulmonary disease (COPD) regardless of any guidance recommendation and of any stage of disease severity, either in fixed dose combination with a long-acting $\beta_{2}$-adrenergic agonist (LABA) or as a component of a triple therapy combination of different inhalers. However, the benefits of ICS in COPD are controversial. There is no recommendation for ICS in Global Initiative for Chronic Obstructive Lung Disease (GOLD) "low-risk" of exarcerbation patients and there are also limitations for those patients at "high-risk". Due to potential severe adverse effects, ICS should be discontinued in patients who do not need them. The safe withdrawal of ICS in COPD constitutes the main thrust of this article. We believe that ICS can be safely withdrawn in patients at low-risk. For patients at high risk of exacerbation, ICS may be discontinued with caution, monitoring changes in forced expiratory volume in one second $\left(\mathrm{FEV}_{1}\right)$ and in peripheral blood eosinophils. In all COPD patients, maintenance therapy with long-acting bronchodilators must be in place. (BRN Rev. 2019;5(1):48-61) Corresponding author: Andrea Rossi, andrea.rossi@aiporicerche.it

Key words: Chronic obstructive pulmonary disease. Inhaled corticosteroids. Long-acting bronchodilators. Severe adverse effects. Withdrawal of inhaled corticosteroids. 


\section{INTRODUCTION}

The appropriate use of inhaled corticosteroids (ICS) in patients with stable chronic obstructive pulmonary disease (COPD) seems an endless debate. This manuscript is not aimed to discuss whether, when, why, and how ICS should or should not be prescribed in COPD ${ }^{1-}$ ${ }^{3}$. Rather, our purpose is to examine the published literature to consider whether ICS can be safely discontinued in COPD patients who do not need them on the basis of the available evidence $^{4,5}$. Interestingly, this is also on its way to become a classic debate ${ }^{6,7}$.

It is well documented that ICS are widely prescribed in patients with stable COPD regardless of any guidance recommendation, across all levels of airflow limitation severity and exacerbation risk ${ }^{5-12}$. It would be reasonable to state that ICS are overprescribed in COPD patients ${ }^{8-12}$. Furthermore, even in the absence of evidence, triple therapy, the combination of different inhalers of long-acting bronchodilators with ICS, is commonly used in clinical practice ${ }^{13,14}$.

In fact, following the basic ethics of medicine, patients should not take useless medications, particularly if it could be potentially dangerous $^{1,2,15,16}$. It has been recognised that the regular use of ICS can be associated with significant and undesired side effects ${ }^{1,2}$. In particular, it has been shown that fluticasone can increase the risk of pneumonia ${ }^{17-19}$. Therefore, the combination of a widespread, potentially inappropriate prescription of ICS with the evidence of significant adverse effects has fueled the interest in the need for ICS withdrawal in COPD ${ }^{6,7,20,21}$. In table 1 , the most common side effects associated with the regular use of ICS in COPD are listed.
TABLE 1. Adverse effects of ICS in COPD ${ }^{1,2}$.

$$
\begin{aligned}
& \text { Increased risk of bone fracture } \\
& \text { Increased risk of pneumonia } \\
& \hline \text { Increased risk of tuberculosis } \\
& \hline \text { Increased risk of cataract } \\
& \hline \text { Skin thinning/easy bruising } \\
& \hline \text { Oropharyngeal candidiasis } \\
& \hline
\end{aligned}
$$

COPD: chronic obstructive pulmonary disease; ICS: inhaled corticosteroids.

\section{BACKGROUND}

Although monotherapy with ICS in COPD patients has been reported in clinical practice ${ }^{8}$, it is not recommended by any guidance or consensus document. Likewise, the association of ICS with long-acting muscarinic antagonist (LAMA) is rare and neither supported by any clinical trials nor recommended by any document on COPD, at least by now. Therefore, the focus of our article is on the large number of COPD patients who are on maintenance therapy either with the association of ICS with long-acting $\beta_{2}$-adrenergic agonist (LABA), most often in the fixed dose combination (FDC) ICS/LABA, or on triple therapy.

To our knowledge, the international and national guidance and consensus documents COPD management agree on two points. First, long-acting bronchodilators are the cornerstone of regular pharmacotherapy, either in monotherapy (with LAMA being preferred to LABA, in general) or in combination/association. Second, ICS represent an add-on treatment of choice for patients with exacerbations. There are many national and international publications ${ }^{22}$, but little doubt can exist that the publications from the Global Initiative for Chronic Obstructive Lung Disease (GOLD) are by far the most popular 
international reference strategy for the management of $\mathrm{COPD}^{23-26}$.

Initially, it was recommended to add ICS on top of the long-acting bronchodilators in COPD patients with a forced expiratory volume in one second $\left(\mathrm{FEV}_{1}\right)<50 \%$ predicted and repeated exacerbations, meaning "three episodes in the last three years" ${ }^{\prime 24}$. Then, after the Evaluation of COPD Longitudinally to Identify Predictive Surrogate End-points (ECLIPSE) publication ${ }^{27}$, repeated was replaced by frequent, meaning $\geq 2$ exacerbations in the preceding year ${ }^{25}$, even regardless of the $\mathrm{FEV}_{1}$ percent predicted value ${ }^{26}$. Although with well-known limitation, the definition of exacerbation was, and is, purely clinical, i.e. "worsening of symptoms requiring change in medication, either inhaled (mild) or systemic (moderate), or hospitalisation (severe)"28,29. Therefore, at present, we can consider appropriate the prescription of ICS in COPD patients with two or more exacerbations and/or one hospitalisation per year. For the patients not matching these inclusion criteria, the withdrawal of ICS should be considered ${ }^{3}$. However, it was stated that "withdrawal studies provide equivocal results regarding the consequence of withdrawal on lung function, symptoms, and exacerbations" ${ }^{\prime \prime 3,26}$. Our purpose, in writing this article, is to investigate whether any reasonable conclusions can be drawn from those "equivocal results".

\section{CLINICAL STUDIES}

Two large randomised controlled trials (RCTs) included some COPD patients with moderate airflow limitation: the Effect of discontinuation of inhaled corticosteroids in patients with chronic obstructive pulmonary disease $(\mathrm{COPE})^{30}$ and the Withdrawal of inhaled corticosteroids in people with COPD in primary care (WISP) ${ }^{31}$ studies. They both concluded that withdrawal of ICS was associated with increased risk of exacerbations. However, none of the two arms was on maintenance therapy with long-acting bronchodilators, and the ICS withdrawal arm was on placebo. Therefore, although of scientific value, these trials cannot be considered of actual clinical interest and will not be discussed further.

Two studies had a prospective "real-life" protocol, i.e. the Real-Life study on the appropriateness of treatment in moderate COPD patients (OPTIMO) ${ }^{32}$ and the Outpatient care with long-acting bronchodilators: COPD registry in Germany (DACCORD) ${ }^{33}$ trials. Four studies can be classified as prospective RCTs, namely Indacaterol: Switching non-exacerbating patients with moderate COPD from salmeterol/fluticasone to indacaterol (INSTEAD) ${ }^{34}$, COPD and Seretide: a Multicenter Intervention and Characterisation (COSMIC) ${ }^{35}$, Withdrawal of Inhaled Steroids During Optimized bronchodilator Management (WISDOM) ${ }^{36}$, and the Study to UNderstand the Safety and Efficacy of ICS withdrawal from Triple therapy in COPD (SUNSET) ${ }^{37}$.

Although all these RCTs enrolled patients with stable COPD, the inclusion criteria varied significantly among protocols, such that they must be analysed separately. In particular, four of them ${ }^{32-34,37}$ recruited patients who may be defined as "low-risk", i.e. with mild-to-moderate airflow limitation and absence of exacerbations, whereas the other two ${ }^{35,36}$ recruited "high-risk" patients, i.e. with more severe airflow limitation and history of at least one moderate-to-severe exacerbation in the year preceding the study. Calzetta et al. ${ }^{20}$ performed a meta-analysis after identification of 
the appropriate studies on PubMed and google scholar concluding that further well-designed studies on withdrawal of ICS should be performed by clustering COPD patients with regard to different phenotypes.

For the purpose of our article, we decided to discuss separately the studies not on the basis of their methodology, i.e. real-life versus RCT, but according to the patients' characteristics, i.e. "low-risk" versus "high-risk", as suggested by the more recent GOLD strategic documents ${ }^{25,26}$.

\section{“LOW-RISK” PATIENTS}

This definition includes patients with a spirometric diagnosis of $\mathrm{COPD}$, i.e. $\mathrm{FEV}_{1} /$ forced vital capacity $(\mathrm{FVC})<0.7$, and with mild-to-moderate airflow limitation, i.e. $\mathrm{FEV}_{1}>50 \%$ predicted, and $<2$ exacerbations in the previous year. Exacerbation, defined by a change in symptoms leading to a change in medication, is classified as mild (inhaled medications changed by the patient), moderate (short-course of antibiotics or systemic corticosteroids or both), and severe (leading to hospitalisation). In these low-risk patients, ICS are not recommended by any guidance document. Therefore, if prescribed, the withdrawal can be considered.

\section{OPTIMO Study: The real-life study On the aPpropriaTeness of treatment In MOderate COPD patients}

The OPTIMO trial is a one country prospective real-life study ${ }^{32}$. It is not an observational study since a change in medication was considered by the design. 914 patients, with a spirometric confirmed diagnosis of COPD, in stable conditions, who were on regular treatment with ICS and LABA, either in FDC or different inhalers, met the inclusion criteria. The decision whether to maintain or to withdraw the ICS treatment was left to the attending physician, who was adequately informed on the content of the GOLD reports at the start-up meeting of the study. Of the 816 patients who completed the 6-month period of observation, $482(59 \%)$ continued with the ICS/LABA treatment, whereas 336 (41\%) had their ICS component withdrawn. The vast majority $(91 \%)$ of these patients were switched to regular treatment with long-acting bronchodilators, while a small minority (9\%) received theophylline and/ or short-acting bronchodilators. In fact, the characteristics of the two groups, i.e. continuation or discontinuation of ICS, did not differ for any of the considered variables at baseline. At the end of the 6-month observational period, there was no significant difference in $\mathrm{FEV}_{1}$ percent predicted, COPD Assessment Test (CAT) or exacerbation rate between the two arms, i.e. ICS continuation or discontinuation (Fig. 1). Although the lack of randomisation was a major limitation of this real-life study, the OPTIMO trial provides observational evidence that in COPD patients with moderate airflow limitation and without exacerbations, ICS can be safely discontinued without increasing the risk of exacerbation, provided that adequate bronchodilator treatment is left in place.

\section{INSTEAD Study: A randomised switch trial of indacaterol versus salmeterol/fluticasone in moderate COPD}

The results of OPTIMO were confirmed by the INSTEAD trial, aimed to demonstrate the 


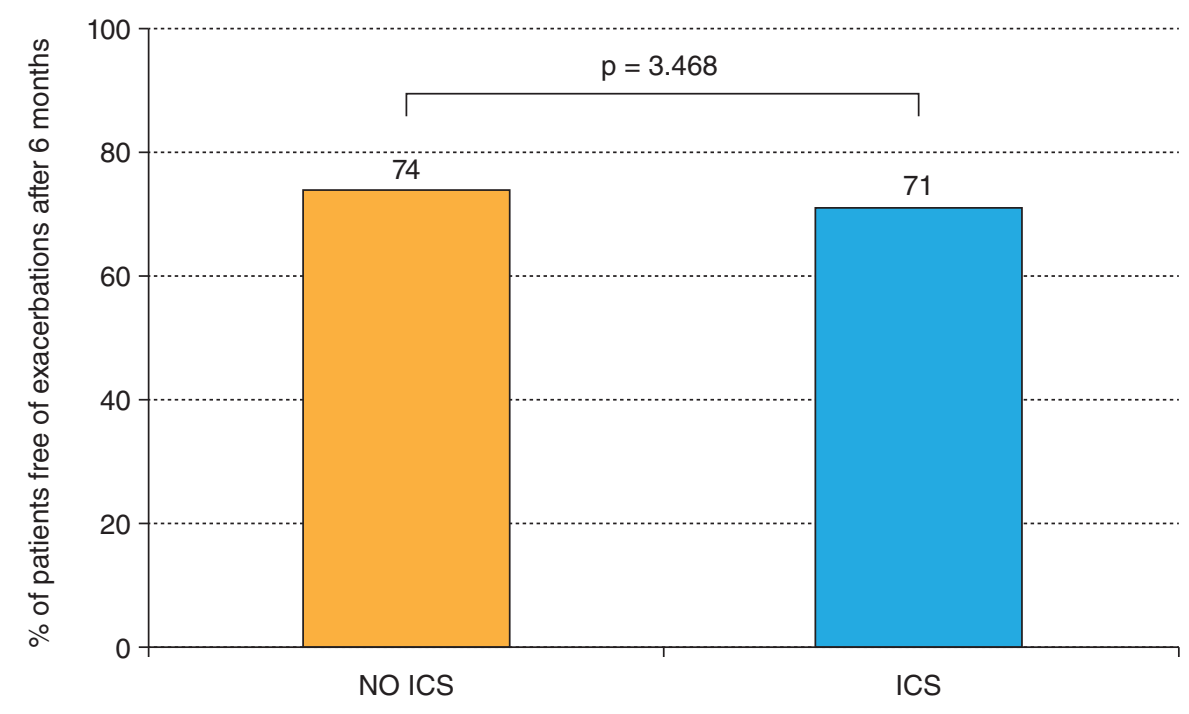

FIgUre 1. Percentage of COPD patients without exacerbations at the end of the six-month period of observation (reproduced with permission from Rossi $A$ et al. ${ }^{32}$ ).

COPD: chronic obstructive pulmonary disease; ICS: inhaled corticosteroids.

non-inferiority of a regular indacaterol therapy (LABA) versus the salmeterol/fluticasone (SFC) (LABA/ICS) treatment in COPD patients at low risk of exacerbation ${ }^{34}$. 581 stable patients, who were on regular treatment with SFC 50/500 mcg b.i.d. dry powder inhaler from at least three months before recruitment, were randomly assigned either to continue their SFC therapy or to be switched to indacaterol 150 mcg q.d. 250 and 246 patients completed the study in the SFC or indacaterol arm respectively. At the end of the 6-month period of observation, there was no difference for the primary end-point, i.e. $\mathrm{FEV}_{1}$ percent predicted at 12 weeks, nor for the secondary end-points, i.e. symptoms, quality of life, and exacerbations (any severity) (Fig. 2). During this trial, two patients reported pneumonia in the SFC group compared to none in the indacaterol arm. The INSTEAD study had two limitations: first it was powered on the $\mathrm{FEV}_{1}$ and not on exacerbations; second, the observational period lasted only six months while many investigators believe that only an observational duration of at least one year can allow to draw solid conclusions on exacerbations $^{20}$. However, the data from the OPTIMO and the INSTEAD studies substantiate each other. Furthermore, although not an ICS discontinuation study, the Efficacy and safety of once-daily QVA149 compared with twice-daily salmeterol/fluticasone in patients with chronic obstructive pulmonary disease (ILLUMINATE) trial ${ }^{38}$ recruited and randomised low-risk COPD patients to receive either the LABA/LAMA combination indacaterol/glycopyrronium 110/50 mcg q.d. or SFC 50/500 mcg b.i.d. In the LABA/LAMA arm, $30 \%$ of 


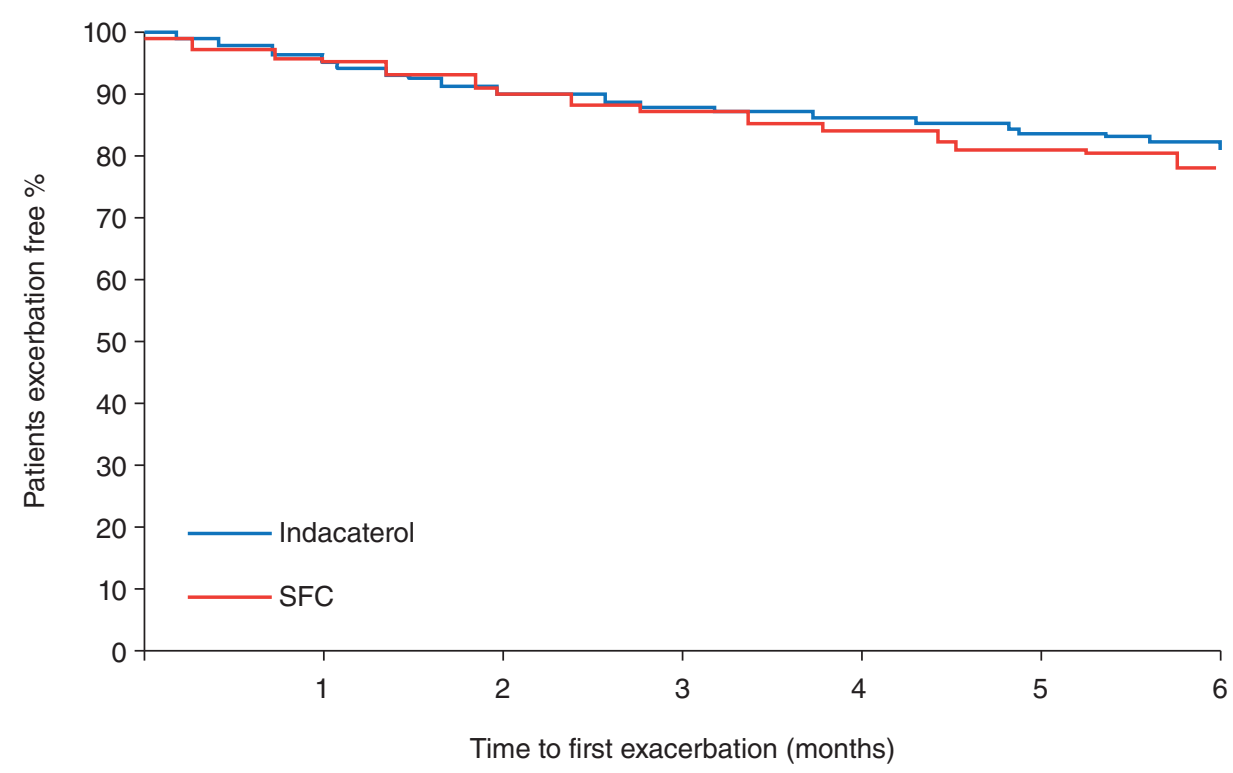

Figure 2. Time to first moderate or severe COPD exacerbation up to week 26. SFC: salmeterol/fluticasone $50 / 500 \mathrm{mcg}$ fixed-dose combination (reproduced with permission of the (C) ERS 2018 from Rossi $A$ et al. ${ }^{34}$ ).

COPD: chronic obstructive pulmonary disease; SFC: salmeterol/fluticasone.

patients were withdrawn from previously prescribed ICS during the washout phase. In that sub-analysis, no differences between groups were seen in outcomes.

\section{DACCORD Study: Outpatient care with long-acting bronchodilators: COPD registry in Germany}

More recently, a real-life sub-group analysis was performed from the DACCORD population in Germany ${ }^{33}$. From the 6122 COPD patients originally enrolled, more than 1000 were receiving ICS prior to entering the study. Following the decision of the attending physicians, 1022 patients continued their ICS regimen for the 2-year follow-up period, whereas 236 patients discontinued the ICS entering the study and did not reinitiate during the 2 years. The two populations were similar at the beginning of the study, although the ICS withdrawal patients had a shorter duration of the disease, and a slightly better lung function: $\mathrm{FEV}_{1}$ percent predicted $67.4 \%$ versus $59.8 \%$ in the ICS withdrawal and ICS continuation group, respectively. However, neither an increase in exacerbations nor deterioration in health status was observed in the group discontinuing the ICS treatment. The annualised exacerbation rate averaged 0.414 and 0.433 in the withdrawal and continuation ICS group, respectively, without differences in the prevalence of exacerbating patients which averaged $74.2 \%$ and $70.7 \%$, respectively, before the entry into the study. This 2-year real-life study concluded that ICS withdrawal is possible with no increased risk of exacerbation in 
patients with COPD managed in the primary and secondary care. It should be noted that in these populations, $62 \%$ of patients had a $\mathrm{FEV}_{1} \geq 50 \%$ predicted and about $70 \%$ of patients, without differences between the groups, did not report exacerbations during the six months prior to entry. Hence, the COPD population of the DACCORD study can be considered rather representative of the general COPD population in real-life primary and secondary care settings. Of note that the attending physicians were more at ease to withdraw ICS in patients with less severe and less established disease with a negligible influence from the level of symptoms and exacerbation history.

\section{SUNSET Study: Study to UNderstand the Safety and Efficacy of ICS withdrawal from Triple therapy in COPD}

In this most recent RCT, SUNSET, after a runin period of triple therapy for one month, 456 and 472 patients completed the 26 weeks treatment with indacaterol/glycopyrronium 110/50 $\mathrm{mcg}$ and tiotropium $18 \mathrm{mcg}$ plus SFC $50 / 500 \mathrm{mcg}$, respectively ${ }^{37}$. The $\mathrm{FEV}_{1} / \mathrm{FVC}$ had to be less than 0.7 and $\mathrm{FEV}_{1}>40 \%$ and $<80 \%$ predicted (post-bronchodilator $\mathrm{FEV}_{1}$ $56.6+10 \%$ predicted), without frequent exacerbations: $66 \%$ and $34 \%$ of patients had 0 and 1 exacerbation in the previous year respectively. The primary end-point of a non-inferiority decline in post-dose through $\mathrm{FEV}_{1}$ was missing. In fact, the mean $\mathrm{FEV}_{1}$ was almost $30 \mathrm{ml}$ lower, on average, in the dual therapy group with the lower limit exceeding the $-50 \mathrm{ml}$ selected as a threshold. The difference was even greater in patients with peripheral blood eosinophil count $>2 \%$ and 300 cell $/ \mu \mathrm{L}$. These patients had also a greater rate of moderate-to-severe exacerbations and a shorter time to first moderate-to-severe exacerbation. By contrast, the analysis of the overall patient population did not show any difference for exacerbation rate nor for time to first exacerbation between the two arms.

\section{Comment}

In summary, the data from these studies sustain similar conclusions for the low-risk COPD patients. The discontinuation of ICS does not cause a recrudescence of exacerbations in the vast majority of patients. There are some limitations to be considered: lack of randomisation ${ }^{32,33}$, short duration $32,34,37$, posthoc sub-group analysis ${ }^{33,38}$. Furthermore, the two RCTs, INSTEAD ${ }^{34}$ and SUNSET ${ }^{37}$, did not have exacerbations as the primary end-point. However, no deterioration in lung function, symptoms, and exacerbation rate was observed in three studies ${ }^{32-34}$ after withdrawal of ICS, provided that maintenance treatment with long-acting bronchodilators was in place. In the SUNSET study, a small but stable decrease in $\mathrm{FEV}_{1}$ was observed, and more action was recommended for patients with peripheral blood eosinophils. The latter issue was not examined in the other three studies. However, it seems that monitoring of $\mathrm{FEV}_{1}$ is a wise strategy when changing therapy in COPD patients.

\section{“HIGH-RISK” PATIENTS}

Four large clinical trials addressing the issue of ICS withdrawal, including an active 
comparator, have been published. The INSTEAD $^{34}$ and the SUNSET ${ }^{37}$ have been considered in the previous section on low-risk patients. Two studies dealt with COPD patients at higher risk of exacerbations and are discussed below.

\section{COSMIC Study: COPD and Seretide: a Multicenter Intervention and Characterization}

The COSMIC trial ${ }^{35}$ was published more than 10 years ago and explored the consequences of ICS withdrawal in COPD patients with moderate-to-severe airflow limitation $\left(\mathrm{FEV}_{1}\right.$ $30-70 \%$ predicted, although the mean $\mathrm{FEV}_{1}$ was $46 \%$ predicted) and $\geq 2$ exacerbation in the previous year. After a 3-month run-in period during which 373 patients received SFC 50/500 mcg b.i.d., 189 patients were randomised to continue the SFC treatment whereas 184 patients were randomised to discontinue the fluticasone medication and remained on maintenance therapy with salmeterol. 155 and 138 patients concluded the one-year study in the SFC or salmeterol arm, respectively. No significant difference was found between the groups in the annual rate of moderate or severe exacerbations. The severity of airflow limitation was the best predictor of moderate-to-severe exacerbation, regardless of ICS treatment. However, rates of mild exacerbations ( $\geq 3$ extra inhalations of rescue medication with short-acting bronchodilators per day on $\geq 2$ consecutive days) were greater in the SFC discontinuation group than in the ICS continuation group.

Significant differences were observed for some secondary outcomes. In the SFC discontinuation arm, the salmeterol group experienced a higher use of rescue medication, more dyspnoea and disturbed nights. Furthermore, the mean $\mathrm{FEV}_{1}$ declined more rapidly in the ICS withdrawal group such that, at the end of the one-year study, it was about $50 \mathrm{ml}$ lower than in the SFC arm. Pneumonia rates were not reported.

In conclusion, the COSMIC trial discourages ICS withdrawal in severe COPD patients with frequent exacerbations, who are on maintenance treatment with the ICS/LABA (FDC), to prevent deterioration in lung function and in patients' related outcomes as well as a higher risk of mild exacerbations.

\section{WISDOM Study: Withdrawal of Inhaled Steroids During Optimized bronchodilator Management}

More recently, the WISDOM study planned a different approach ${ }^{36}$. First the selected COPD patients had to have severe-to-very-severe airflow limitation (i.e. $\mathrm{FEV}_{1}<50 \%$ predicted; mean $\mathrm{FEV}_{1}, 34 \%$ predicted) and $\geq 1$ exacerbation in the previous year being on regular treatment with long-acting bronchodilators (LAMA and/or LABA) and/or ICS or various combinations of LAMA, LABA, ICS. After a 6-week run-in receiving triple therapy (i.e. 18 mcg tiotropium q.d., SFC 50/500 mcg b.i.d.), more than 4000 patients were randomised to either continue the triple therapy or discontinue the ICS and remain on the tiotropium $18 \mathrm{mcg}$ plus salmeterol $50 \mathrm{mcg}$ b.i.d. regimen. Second, at variance from any previous study, the ICS withdrawal was not abrupt, but completed in three steps over 12 weeks ${ }^{39}$. After 52 weeks of observation there was no 


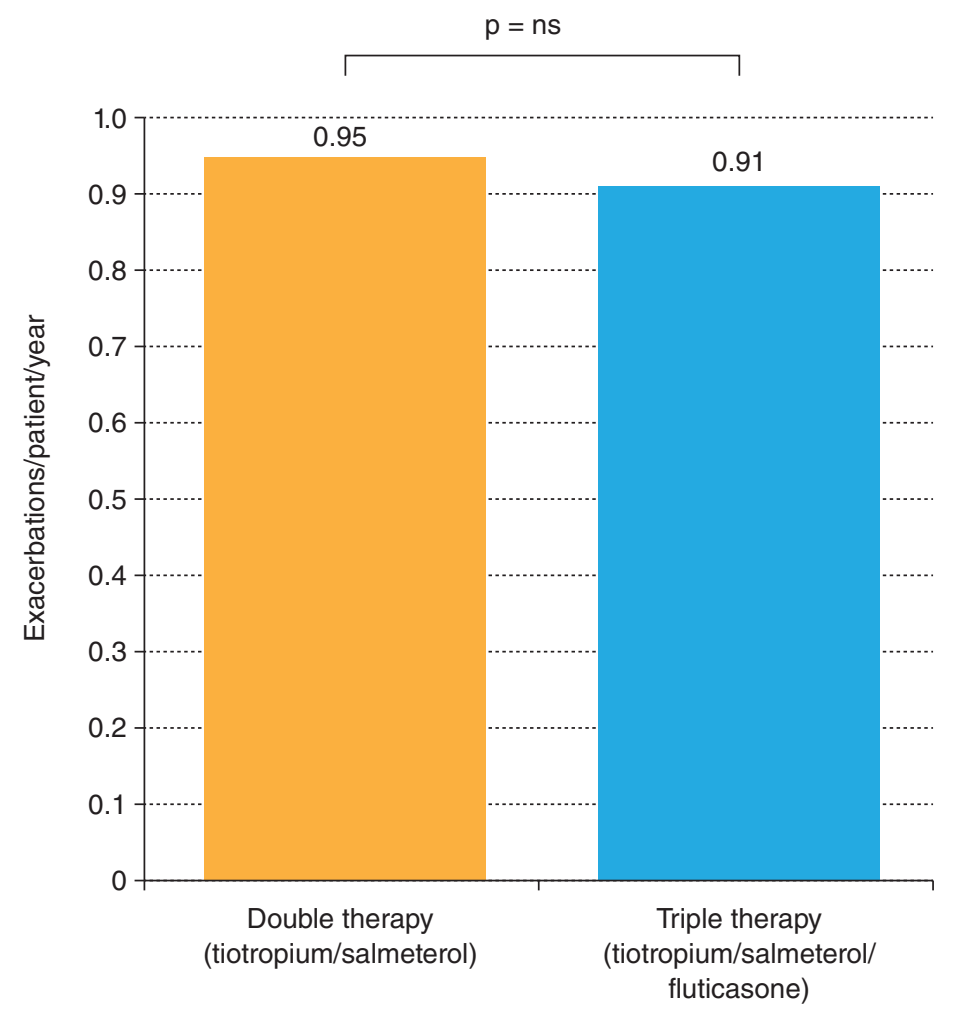

Figure 3. Frequency of exacerbations per patient per year in two groups of patients after fluticasone suspension (double therapy) or continuation (triple therapy). Data from the Withdrawal of Inhaled Steroids During Optimized bronchodilator Management (WISDOM) Study.

significant difference between the two arms for the risk of moderate-to-severe exacerbations and dyspnoea with only a minor change in quality of life. The primary end-point was met: no difference between groups for the time to first moderate exacerbation, i.e. 110 versus 107 days in the withdrawal and continuation arms, respectively. The adjusted exacerbation rate averaged 0.95 and 0.91 per patient per year, respectively (Fig. 3). However, the mean decrease of $\mathrm{FEV}_{1}$ was $-38 \mathrm{ml}$ and $-43 \mathrm{ml}$ greater at week 18 and 52, respectively, in the ICS withdrawal arm than in the triple therapy arm. The small but significant fall in $\mathrm{FEV}_{1}$ observed during the first year of the study was not associated with symptoms and did not progress in the 40 weeks after complete withdrawal of ICS (Fig. 4) ${ }^{40}$. No difference was found between the two arms for the pneumonia rates which amounted to $5.8 \%$ and 5.5\% in the ICS continuation and discontinuation arms, respectively.

\section{Comment}

Although the characteristics of the high-risk COPD patients recruited in the COSMIC and WISDOM trials are somehow different, the results are not so far apart. In fact, there was 
Fluticasone propionate dose

$\square 500 \mu \mathrm{g}$ twice daily $\square$ Reduced to $100 \mu \mathrm{g}$ twice daily $\square$ Reduced to $0 \mu \mathrm{g}$ (placebo)


$-0.12$

Patients $n$

ICS withdrawal

ICS

992941945925913885945918901896868868881876877865850847854840818832839822819814808801797789794806804770793775771768753778763753751745705573 932961960939930908946928921892877854866868853846834839817807781797796807796792789774770778776768765760764742740732739753748736733714688560

Figure 4. Adjusted mean \pm SE change from baseline in on-treatment weekly mean FEV 6 weeks before and 40 weeks after complete ICS withdrawal (reproduced with permission of the (C) ERS 2018 from Magnussen $\mathrm{H}$ et al. ${ }^{40}$ ).

$\mathrm{FEV}_{1}$ : forced expiratory volume in one second; ICS: inhaled corticosteroids.

no significant difference for the occurrence of moderate-to-severe exacerbations in either study whereas there was a significant deterioration of lung function in the ICS withdrawal arm in both trials. Similar results were reported after steroid withdrawal in the Corticosteroids in obstructive lung disease (GLUCOLD) study ${ }^{41}$.

These data are very interesting because of their implication for the guidance reports. In fact, the major, if not unique, motivation to recommend ICS, as add-on treatment on top of long-acting bronchodilators in stable COPD, is the prevention of exacerbation and not the avoidance of lung function loss ${ }^{23-26}$. In view of these data, the rationale to recommend addition of ICS for COPD patients could change and not be based on exacerbations. The mechanisms by which ICS can improve $\mathrm{FEV}_{1}$ in COPD remain poorly understood. The hypothesis goes from the reduction of bronchial wall oedema to the reduction of the release of inflammatory mediators or the enhancement of the $\beta_{2}$-adrenergic smooth muscle relaxing action ${ }^{42}$.

When the results of all the trials, on low- and high-risk patients are considered together only in the COSMIC $^{35}$ study there was a greater risk 
for mild exacerbations and by no means a greater risk for moderate-to-severe exacerbations.

\section{EOSINOPHILS IN PERIPHERAL BLOOD}

Recent work suggests that ICS are more active to prevent exacerbations in COPD patients with high eosinophil counts ${ }^{43,44}$, although with contradicting results ${ }^{45}$. For the presentation of this review, the relevant publication is the post-hoc analysis from the WISDOM RCT by Watz et al. ${ }^{46}$. They found that in the 2296 COPD patients with peripheral blood eosinophil count, receiving treatment after ICS withdrawal, the exacerbation rate was affected by either the percentage or the absolute eosinophil cutoffs. Patients with $\geq 4 \%$ or $\geq 300$ cells per $\mu \mathrm{L}$ experienced a greater exacerbation rate in the ICS withdrawal arm than in the ICS continuation arm. It was concluded that ICS withdrawal can have a deleterious effect in COPD patients with severe airflow limitation, at least one moderate-to-severe exacerbation in the preceding year, and high peripheral blood eosinophilic count ( $4 \%$ or greater or $\geq 300$ cell $/ \mu \mathrm{L}$ ). Harlander et al. ${ }^{21}$ observed that with the change in COPD classification of severity from GOLD 2013 to GOLD 2017, many patients would move from group D to group B. In fact, the GOLD 2017 classification did not take into account any more the severity of airflow limitation as a factor increasing, per se, the risk of exacerbations. The authors ${ }^{21}$ conclude that withdrawal of ICS would be feasible in the group of patients moving from group $D$ to $B$ with the exclusion of patients with a suspected asthma-COPD overlap (ACO) and those with high peripheral blood eosinophils, suggesting $\geq 300$ cells $/ \mu \mathrm{L}$ as a cut-off count.

\section{META-ANALYSIS}

Calzetta et al. ${ }^{20}$ performed a large and rigorous meta-analysis on the issue of withdrawal of ICS in COPD. They found that ICS withdrawal did not increase the overall rate of exacerbations, although the time to first exacerbation was slightly but significantly shorter in the group of patients discontinuing ICS. They also reported a significant deterioration in lung function, averaging $-30 \mathrm{ml}$ (from -42 to $-18 \mathrm{ml}$ in $\mathrm{RCT}$ ), and in health quality of life, although the slight increase $(+1,24$ Saint George Respiratory Questionnaire units, on average) did not approach the clinical significant threshold. One might argue that a loss in $\mathrm{FEV}_{1}$ in patients with severe airflow limitation should not be permitted. However, the range goes from $-40 \mathrm{ml}$ to $-12 \mathrm{ml}$. First, these data show a high inter-individual variability, with patients losing a negligible portion of their $\mathrm{FEV}_{1}$. Second, frequent measurement of $\mathrm{FEV}_{1}$, which can be performed almost daily at home with telemonitoring by implementation of modern technology, can help to detect the faster decliner, such that ICS will not be discontinued only in these patients and not in all. This is important in view of the adverse effects of ICS in COPD. Finally, one would argue whether an average improvement of $+30 \mathrm{ml}$ would be stressed as sufficient to prescribe a new bronchodilator in COPD, even with severe airflow limitation.

Although of undoubted scientific value, the meta-analysis by Calzetta et al. ${ }^{20}$ takes into account the difference between observational studies and RCTs but does not differentiate the "level of risk", such that the studies on GOLD low-risk and high-risk patients are analysed together. We understand that the number of 
studies is too small to allow various different analyses. However, we believe that the issue of ICS withdrawal has different weight in lowrisk patients, for whom there is no recommendation compared to high-risk patients for whom such recommendation exists. The conclusion in the article by Calzetta et al. ${ }^{20}$ sounds wise and is worth sharing. There is a need of additional studies by clustering the patients with regard to phenotype, rate of exacerbations, lung function decline, and quality of life. However, this kind of studies should focus on COPD patients with frequent exacerbations and/or high peripheral blood eosinophils, not on the large population of low risk patients for whom the lack of recommendation is associated with evidence of safe withdrawal when long-acting bronchodilator therapy is in place.

Along these lines, it should be noted that several factors can influence the relapse after discontinuation of ICS in COPD in general practice $^{47}$. Sex, age, smoking habits, and reversibility of airflow limitation might predict a less favorable outcome. However, these conclusions come from a small observational study and need further investigation.

\section{CONCLUSIONS}

In view of the large number of COPD patients receiving inappropriate prescription of ICS/ LABA or even triple therapy, the withdrawal of ICS from maintenance therapy deserves great attention, in view of the long-term safety issue associated with the ICS use and abuse (see Table 2). For a long time and under many circumstances, COPD has been classified as an "inflammatory disorder of the airways". Although some role of inflammation cannot
TABLE 2. Withdrawal of ICS in COPD

Safe: patients with mild-to-moderate airflow limitation, less than 2 exacerbations in the previous year, less than 300 eosinophils $\mu \mathrm{L}$ in the peripheral blood.

With caution: severe airflow limitation with less than 2 exacerbations in the previous year and careful documentation of more than 300 eosinophils $\mu \mathrm{L}$ in peripheral blood; monitoring of lung function is recommended.

Not at present: severe airflow limitation, and more than 1 exacerbation in the previous year, and careful documentation of more than 300 eosinophils $\mu \mathrm{L}$ in the peripheral blood.

PROS and CONS

PROS of ICS withdrawal in COPD: less medications to be assumed by the patient; prevention of all the adverse effects due to ICS in COPD, in particular osteoporosis and pneumonia.

CONS of ICS withdrawal in COPD: possible deterioration of lung function in patients with severe airflow limitation; potential increased risk of exacerbations in patients with history of exacerbations and eosinophilia.

COPD: chronic obstructive pulmonary disease; ICS: inhaled corticosteroids.

be discharged, the pathogenesis of COPD is much more complex and different from asth$\mathrm{ma}^{48-50}$. Therefore, the small benefit of ICS in a limited number of patients is not surprising. Provided that the maintenance therapy with long-acting bronchodilators is in place and that the patient takes the prescribed medications, ICS withdrawal should be solidly considered in all patients with $<2$ exacerbations/ year. Frequent measurement of $\mathrm{FEV}_{1}$ helps to prevent excessive deterioration in lung function. Although the evidence is limited, a solid count of peripheral blood eosinophils recommends caution in the discontinuation of ICS, which should not be undertaken if a history of exacerbation is present.

\section{DISCLOSURES}

Dr. Andrea Rossi has received fees for consultation and lectures and support for meetings 


\section{from Chiesi, Novartis, Boehringer Ingelheim, TEVA. Dr. Erika Zanardi has received support for participation in scientific meetings from Menarini, Guidotti-Malesci.}

\section{Dr. Andrea Rossi and Dr. Erika Zanardi re- port that the reports and personal fees have no bearing on the work that is presented in the body of the manuscript.}

\section{REFERENCES}

1. Price D, Yawn B, Brussels G, Rossi A. Risk-to-benefit ratio of inhaled corticosteroids in patients with COPD. Prim Care Respir J. 2013;22:92-100.

2. Rossi AP, Zanardi E, Zamboni M, Rossi A. Optimizing treatment of elderly COPD patients: what role for inhaled corticosteroids? Drugs Aging. 2015; 32:679-87.

3. Vogelmeier CF, Criner GJ, Martinez FJ. Global Initiative for Chronic Obstructive Lung Disease (GOLD) Revisions 2001-2017: Historical and Critical Perspective. BRN Rev. 2017:3:151-65.

4. Suissa S, Rossi A. Weaning from inhaled corticosteroids in COPD: the evidence. Eur Respir J. 2015:46:1232-5.

5. Yawn B, Suissa S, Rossi A. Appropriate use of inhaled corticosteroids in COPD: the candidates for safe withdrawal. NPJ Prim Care Respir Med. 2016; 26:16068

6. Chalmers JD. POINT: should an attempt be made to withdrawal inhaled corticosteroids in all patients with stable GOLD $330 \%<\mathrm{FEV}_{1}<50 \%$ predicted) COPD? YES. Chest. 2018;153;778-82.

7. Pavord ID. COUNTERPOINT: should an attempt be made to withdrawal inhaled corticosteroids in all patients with stable GOLD $330 \%<$ FEV $1<50 \%$ predicted) COPD? NO. Chest. 2018;153;782-7.

8. Corrado A, Rossi A. How far is real life from COPD therapy guidelines? An Italian Observational study. Respir Med. 2012;106:989-97.

9. Roche N, Pribil C, Van GE et al. Real-life use of fluticasone propionate/ salmeterol in patients with chronic obstructive pulmonary disease: a French observational study. BMC Pulm Med. 2014;14:56.

10. Price D, West D, Brussels G et al. Management of COPD in the UK primary-care setting: an analysis of real-life prescribing patterns. Int J Chronic Obstruct Pulm Dis. 2014; 9:889-905.

11. Chalmers JD, Tebboth A, Ternouth A, Ramscar N. Determinants of inhaled corticosteroids use with GOLD A/B COPD: a retrospective study of UK general practice. NPJ Prim Care Respir Med. 2017;27:43.

12. Worth, Buhl R, Criée C et al. GOLD 2017 treatment pathways in "real life": an analysis of DACCORD observational trial. Respir Med. 2017;131:77-84.

13. Brusselle G, Price D, Gruffyd-Jones $\mathrm{K}$ et al. The inevitable drift to triple therapy in COPD: an analysis of prescribing pathways in the UK. Int J Chronic Obstruct Pulm Dis. 2015:10:2207-17.

14. Papi A, Vesto J, Fabbri LM et al. Extrafine inhaled triple therapy versus dual bronchodilator in chronic obstructive pulmonary disease (TRIBUTE): a double blind, parallels group, randomized controlled trial. Lancet. 2018; 391:1076-84.

15. Suissa S, Ernst P. Precision medicine urgency: The case of inhaled corticosteroids in COPD. Chest. 2017; 152:227-31.

16. Cho MK, Tantissima KG. Adrenal Insufficiency and ICS: genetics take a breath. Lancet Respir Med. 2018;6:407-8.
17. Calverley PMA, Anderson JA, Celli B et al. Salmeterol and fluticasone propionate and survival in chronic obstructive pulmonary disease. N Eng J Med. 2007;356:775-89.

18. Dransfield MT, Bourbeau J, Jones PW et al. Once daily inhaled fluticasone furcate and vilanterol versus vilanterol only for prevention of exacerbations of COPD: two replicate double-blind, parallel-group, randomized controlled trials. Lancet Respir Med. 2013;1:210-23.

19. Crim C, Dransfield MT, Bourbeau J et al. Pneumonia risk with inhaled fluticasone furcate and vilanterol compared with vilanterol alone in patients with COPD. Ann Am Thor Soc. 2015; 12:27-34.

20. Calzetta L, Matera MG, Braido F et al. Withdrawal of inhaled corticosteroids in COPD: a meta-analysis. Pulmon Pharmacol Therap. 2017;45: 148-58.

21. Harlander M, Barrecheguren M, Turel M, Miravitlles M. Should patients switched from D to B in the GOLD 2017 classification be discontinued from inhaled corticosteroids? COPD. 2017;14:465-8.

22. Miravitlles M, Roche N, Cardoso J et al. Chronic Obstructive pulmonary disease in Europe: a look into the future. Eur Respir J. 2018;19:11.

23. Pauwels RA, Buist S, Calverley PMA et al. Global strategy for the diagnosis, management, and prevention of chronic obstructive pulmonary disease. Am J Respir Crit Care Med. 2001;163:1256-76.

24. Rabe KF, Hurd S, Anzueto A et al. Global strategy for the diagnosis, management, and prevention of chronic obstructive pulmonary disease. Am J Respir Crit Care Med. 2007;176:532-55.

25. Vestbo J, Hurd S, Agustí A et al. Global strategy for the diagnosis, management, and prevention of chronic obstructive pulmonary disease. Am J Respir Crit Care Med. 2013;187:347-65.

26. Vogelmeier CF, Criner GJ, Martinez FJ et al. Global strategy for the diagnosis, management, and prevention of chronic obstructive pulmonary disease. Eur Respir J. 2017;49:1700214.

27. Hurst JR, Vestbo J, Anzueto A et al. Susceptibility to exacerbation in chronic obstructive pulmonary disease. N Eng J Med. 2010;363:1128-38.

28. Rodriguez-Roisin R. Toward a consensus definition for COPD exacerbations. Chest. 2000;117:398S-401S.

29. Celli B. Dissecting COPD exacerbations: time to rethink our definition. Eur Respir J 2017;50:1701432.

30. van der Valk P, Monninkkhof E, van der Palen J et al. Effect of discontinuation of inhaled corticosteroids in patients with chronic obstructive pulmonary disease; the COPE study. Am J Respir Crit Care Med. 2002;166: 1358-63.

31. Choudhurry AB, Dawson CM, Klivington HE et al. Withdrawal of inhaled corticosteroids in people with COPD in primary care: a randomized controlled trial. Respir Res. 2007;8:93.

32. Rossi A, Guerriero M, Corrado A. Withdrawal of inhaled corticosteroids can be safe in COPD patients at low risk of exacerbation: a real-life study on the appropriateness of treatment in moderate COPD patients (OPTIMO). Respir Res. 2014;15:77.

33. Vogelmeier C, Worth H, Buhl R et al. "Real-life" inhaled corticosteroid withdrawal on COPD: a sub-group analysis of DACCORD. Int J Chronic Obstruct Pulm Dis. 2017;12:487-94.

34. Rossi A, van der Molen T, del Olmo R et al. INSTEAD: a randomized switch trial of indacaterol versus salmeterol/fluticasone in moderate COPD. Eur Respir J. 2014;44:1548-56.

35. Wouters EFM, Postma D, Fokkenst B et al. Withdrawal of fluticasone propionate from combined salmeterol/fluticasone treatment in patients with COPD causes immediate and sustained disease deterioration: a randomized controlled trial. Thorax. 2005;60:480-7.

36. Magnussen H, Disse B, Rodriguez-Roisin R et al Withdrawal of inhaled corticosteroids and exacerbation of COPD. N Eng J Med. 2014;371: 1285-94.

37. Chapman KR, Hurst JR, Frent SM et al. Lung-term triple therapy de-escalation to indacaterol(glycopirronium in COPD patients (SUNSET): a randomized, double-blind, triple-dummy clinical trial. Am J Respir Crit Care Med. 2018;198:329-39. 
38. Vogelmeier CF, Bateman D, Pallante J et al. Efficaly and safety of once-adaily QVA149 compared with twice -daily salmeterol-fluticasone in patients with chronic obstructive pulmonary disease (ILLUMINATE): a randomized double-blind, parallel group study. Lancet Respir Med. 2012;1:51-60.

39. Magnussen H, Watz H, Kirsten et al. Stepwise withdrawal of inhaled corticosteroids in COPD receiving dual bronchodilation: WISDOM study design and rationale. Respir Med. 2014; 108:593-9.

40. Magnussen H, Tetzalaff K, Bateman ED et al. Lung function changes over time following withdrawal of inhaled corticosteroids in patients with severe COPD. Eur Respir J. 2016;47:651-4.

41. Kunz LIZ, Postma DS, Klooster K et al. Relapse in FEV1 decline after steroid withdrawal in COPD. Chest. 2015;148:389-96.

42. Rodriguez-Roisin R, Arismendi E. Inhaled corticosteroids withdrawal in severe patients with chronic obstructive pulmonary disease: a wisdom decision? Arch Bronconeumol. 2015;51:57-8.

43. Pascoe S, Locantore N, Dransfield MT, Barnes NC, Pavor ID. Blood eosinophil count, and response to the addition of inhaled fluticasone furcate to villanterol in patients with chronic obstructive pulmonary disease: a secondary analysis of data from two parallel randomized controlled trials. Lancet Respir Med. 2015;3:435-42.

44. Bafadhel M, Peterson S, De Blas MA et al. Predictors of exacerbation risk and response to budesonide in patients with chronic obstructive pulmonary disease: a post hoc analysis of three randomized trials. Lancet Respir Med. 2018;6:117-26.

45. Roche N, Chapman KR, Vogelmeier CF et al. Blood eosinophils and response to maintenance chronic obstructive pulmonary disease treatment. Data from the FLAME trial. Am J Respir Crit Care Med. 2017;195: 1189-97.

46. Watz H, Tetzeloff K, Wouters EFM et al. Blood eosinophil count and exacerbations in severe chronic obstructive pulmonary disease after withdrawal of inhaled corticosteroids: a post hoc analysis of the WINSDOM trial. Lancet Respir Med. 2016;4:390-8.

47. Shermer TRJ, Hendriks AJC, Chavannes NH et al. Probability and determinants of relapse after discontinuation of inhaled corticosteroids in patients with COPD in general practice. Prim Care Respir J. 2004;13:48-55.

48. Hogg JC, Paré PD, Hackett TL. The contribution of small airway obstruction in the pathogenesis of chronic obstructive pulmonary disease. Physiol Rev. 2017; 97:529-52.

49. Eapen MS, Myers S, Walters HD, Sola SS. Airway inflammation in chronic obstructive pulmonary disease (COPD): a true paradox. Expert Rev Respir Med. 2017; 11:10,827-39.

50. Chilosi M, Carloni A, Rossi A, Poletti V. Premature lung aging and cellular senescence in the pathogenesis of idiomatic pulmonary fibrosis and COPD/ emphysema. Transl Res. 2013; 162:156-73. 